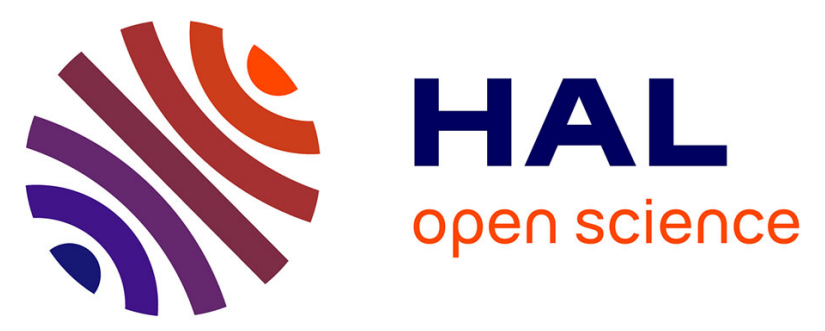

\title{
Monitoring individual cell-signaling activity using combined metal-clad waveguide and surface-enhanced fluorescence imaging
}

\author{
Thomas Söllradl, Kevin Chabot, Ulrike Fröhlich, Michael Canva, Paul \\ Charette, Michel Grandbois
}

\section{To cite this version:}

Thomas Söllradl, Kevin Chabot, Ulrike Fröhlich, Michael Canva, Paul Charette, et al.. Monitoring individual cell-signaling activity using combined metal-clad waveguide and surface-enhanced fluorescence imaging. Analyst, 2018, 143 (22), pp.5559 - 5567. 10.1039/C8AN00911B . hal-01927532

\section{HAL Id: hal-01927532 \\ https://hal.science/hal-01927532}

Submitted on 25 Feb 2021

HAL is a multi-disciplinary open access archive for the deposit and dissemination of scientific research documents, whether they are published or not. The documents may come from teaching and research institutions in France or abroad, or from public or private research centers.
L'archive ouverte pluridisciplinaire HAL, est destinée au dépôt et à la diffusion de documents scientifiques de niveau recherche, publiés ou non, émanant des établissements d'enseignement et de recherche français ou étrangers, des laboratoires publics ou privés. 
Monitoring individual cell-signaling activity using combined metal-clad waveguide and surfaceenhanced fluorescence imaging

Thomas Söllradl ${ }^{\text {abcd }}$, Kevin Chabot ${ }^{\mathrm{ab}}$, Ulrike Fröhlich ${ }^{\mathrm{c}}$, Michael Canva (D)ab, Paul G. Charette ${ }^{\mathrm{ab}}$ and Michel Grandbois (D)*ac

aLaboratoire Nanotechnologies Nanosystèmes (LN2) - CNRS UMI-3463, Université de Sherbrooke, Canada. E-mail: Michel.Grandbois@USherbrooke.ca; Tel: +1 $819821-8000$ ext. 72369 bInstitut Interdisciplinaire d'Innovation Technologique (3IT), Université de Sherbrooke, Canada 'Département de Pharmacologie et Physiologie, Université de Sherbrooke, Canada ${ }^{\mathrm{d}}$ Carinthian Tech Research AG, Villach, Austria

Received 16th May 2018, Accepted 20th September 2018

First published on 10th October 2018

Evanescent field based biosensing systems such as surface plasmon resonance (SPR), diffraction gratings, or metal-clad waveguides (MCWGs) are powerful tools for label-free real-time monitoring of signaling activity of living cells exposed to hormones, pharmacological agents, and toxins. In particular, MCWG-based imaging is well suited for studying relatively thick objects such as cells due to its greater depth of penetration into the sensing medium, compared to SPR. Labelfree methods, however, provide only indirect measurements in that the measured signal arises from local changes in material properties rather than from specific biomolecular targets. In the case of cells, the situation is especially complex as the measured label-free signal may result from a combination of very diverse sources: morphological changes, intra-cellular reorganization, cascaded molecular events, protein expression etc. Consequently, deconvolving the contributions of specific sources to a particular cell response profile can be challenging. In the following, we present a cell imaging platform that combines two distinct sensing modalities, namely label-free MCWG imaging and label-based surface enhanced fluorescence (SEF), designed to facilitate the identification of the underlying molecular and structural contributions to the label-free MCWG images. We demonstrate the bimodal capabilities of this imaging platform in experiments designed to visualize actin cytoskeleton organization in vascular smooth muscle cells. We then monitored the real-time response of HEK293 cells expressing the Angiotensin 1 receptor (AT1R), when stimulated by the receptor agonist Angiotensin II (AngII). The analysis of the simultaneous label-free signal obtained by MCWG and the intracellular calcium signal resulting form AT1R activation, measured by SEF, allows relating label-free signal features to specific markers of receptor activation. Our results show that the intracellular calcium levels normally observed following AT1R activation are not required for the initial burst of cellular activity observed in the MCWG signal but rather indicates signaling activity involving the intracellular kinase ROCK. 


\section{Introduction}

Eukaryotic cells express a wide variety of structural and functional components to fulfill their tasks and maintain tissue and organ functions. For example, the cytoskeleton of smooth muscle cells generates and maintains forces to regulate vascular tone or engage in rhythmic, synchronized contractions. ${ }^{-}$To sustain individual cell activity associated with physiological functions, cells express a wide variety of membrane-bound receptors that respond to a wide variety of external biochemical stimuli. The largest class of such receptors is the G-protein-coupled receptors (GPCRs), which control a variety physiological processes including cell-cell communication, vascular tone, hormonal signaling, and neuronal transmission. ${ }^{2}$ The reliable detection and quantification of GPCR activity is of great interest in the fields of cellular biology and pharmacology.

Cell-based assays using a variety of label-free transduction methods such as diffraction gratings, $\underline{\underline{3}}$ electrical impedance, $\frac{4}{}$ and surface plasmon resonance ${ }^{5-11}$ have been proposed to study cellsignaling with no need for exogenous markers. Recently, a more flexible type of evanescent field transduction based on metal clad waveguides (MCWG) has been used in sensing applications with cells and bacteria to probe deeper into the cell body $\underline{12,13}$ than is possible with SPR (typically 200 $\mathrm{nm}$ or less for visible and near-IR systems). Using appropriate optics, SPR and MCWG can be used for imaging where advanced systems are capable of resolving intracellular structures. Our group and others have demonstrated this capability in a variety of cell-substrate interaction experiments, $\frac{8,14-16}{1 n}$ particular to provide insight into the heterogeneous response of a cell

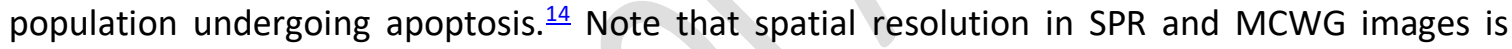
limited along the direction of light propagation by the finite mode attenuation length, rather than by diffraction. $\frac{17,18}{1}$ Furthermore, there is an intrinsic trade-off between mode attenuation length and penetration depth into the sensing medium such that any increase in penetration depth comes at the expense of a commensurate decrease in imaging spatial resolution. Compared to SPR, MCWG offers the better compromise between spatial resolution and penetration depth. $\underline{13}$ Hence, when imaging relatively thick objects such as eukaryotic cells or bacteria, this tradeoff is especially relevant and MCWG-based imaging is preferred.

Since SPR and MCWG are label-free methods, they yield direct information on local changes in material properties (in this case the refractive index), rather than on specific molecular targets. As with all label-free methods, however, it can sometimes be challenging to unambiguously identify molecular targets or cell structures from the image data. Indeed, though various SPRbased imaging experiments have been applied to study cellular structure and functional activity, few studies have attempted to identify the particular subcellular structures involved. $\frac{8,19-22}{\text { Some }}$ of the most successful attempts involve separate corroborating experimental measurements with conventional fluorescence microscopy to identify a-posteriori the cellular features in the labelfree micrographs. $\frac{8,19}{19}$

Image fusion methods attempt to provide the best of both worlds by combining distinct imaging modalities, ideally in a single instrument, such as with simultaneous atomic force and confocal fluorescence microscopy. Evanescent field based methods such as MCWG and SPR are capable of simultaneous label-free and fluorescence imaging, where the evanescent field in the dielectric 
can be used to excite fluorescent markers near the surface in a manner similar to total internal reflection fluorescence (TIRF) microscopy. Furthermore, the resonant coupling to the guided surface mode in MCWG and SPR produces a significant field enhancement in the sensing medium compared to TIRF, as demonstrated with surface plasmon enhanced fluorescence (SPEF). $\frac{23}{}$ Indeed, SPEF has been shown to increase detection sensitivity in classic fluorescence-based biomolecular assays by orders of magnitude. ${ }^{24}$ More recently, our group and others applied SPEF in cell sensing to identify the specific cellular processes underlying an SPR response. $\underline{22,25,26}$

In this work, we introduce the first microscopy platform to combine MCWG and surface-enhanced fluorescence (SEF) imaging, a method we term "MCWG-SEF microscopy". Relative to SPR, the enhanced probing depth of MCWG enables simultaneous label-free and fluorescently-labeled imaging of cellular structures further up into the cell body, while the improved trade-off between resolution and probing depth maximizes the achievable spatial resolution. A first series of validation experiments are presented where actin stress fibers are simultaneously imaged by MCWG and SEF in vascular smooth muscle cells (VSMC). A second series of experiments is presented showing intracellular signaling associated with the activation of AT1R by its agonist Angll in a stably transfected cell model. Intracellular calcium levels, a robust and specific marker of AT1R signaling, are recorded by SEF with the fluorescent probe Fluo8, while simultaneously capturing the whole cell response with MCWG imaging. Use of a pharmacological inhibitor made it possible to correlate specific downstream signaling pathways and heterogeneity in the Anglldependent cell response with the information in the MCWG images.

\section{Materials and methods}

\subsection{MCWG-SEF microscopy platform}

The MCWG-SEF microscopy platform is an expansion on a custom-built MCWG imaging system based on a high-numerical-aperture objective described in detail elsewhere. $\underline{13}$ The sensor chips (MCWG substrate) with the cells under study are placed in a fluid chamber (QR-43C, Harvard Apparatus). As shown in Fig. 1, the light sources are collimated low-coherence fiber-coupled LEDs $\left(\lambda_{\text {nom }}=470 \mathrm{~nm}\right.$ from Thorlabs or $\lambda_{\text {nom }}=530 \mathrm{~nm}$ from Mightex Systems) chosen to minimize diffraction artifacts and phototoxicity. Input light passes through a bandpass filter (530/10 or 470/20, Chroma Tech) and is focused at the back focal plane of a high-numerical-aperture microscope objective $(63 \times, N A=1.46$, Zeiss). The offset of the focused spot from the objective's optical axis determines the incidence angle of the collimated input light at the backside of the

MCWG substrate. The reflected light is collected back through the objective where a polarizing beam-splitter separates each component for visualization by a pair of CCD camera $(1392 \times 1040$, 12 bit, gray-scale, Allied Vision) at an acquisition rate of $1 \mathrm{~Hz}$. As designed, the MCWG sensor chips support a single (fundamental) transverse magnetic (TM) mode and a single (fundamental) transverse electric (TE) mode. The incidence angle of the input light is chosen to couple solely with the TM mode since it provides the best tradeoff between spatial resolution and penetration depth. $\underline{13}$ Thus, only intensity variations in the TM-component of the reflected light contain information regarding refractive index variations in the sample. In general, however, there may also be source-dependent spatial and temporal intensity variations in the TM-based signal. Since the TE-component of the reflected light is totally internally reflected at the glass/metal interface of the MCWG chips and thus does not interact with the sample, it can be used as a reference to 
correct the TM-based signal for source dependent intensity variations. Fluorescence light is collected simultaneously through the top window of the sample chamber using a long-workingdistance microscope objective (50x, 0.55, Mitutoyo Inc.), passes through an emission filter (HQ525/50 from Chroma Tech or LP615 from Zeiss), and is imaged by a cooled CCD camera (1392 $\times 1040,16$ bit, Qimaging). Note that while fluorescence imaging is diffraction-limited, spatial resolution in the MCWG images is anisotropic: resolution is limited along the direction normal to light propagation in the image plane by diffraction and by the finite mode attenuation length in direction of propagation. As stated above, MCWG provides a lower level of anisotropy compared to SPR at deeper probing depths. $\underline{13}$

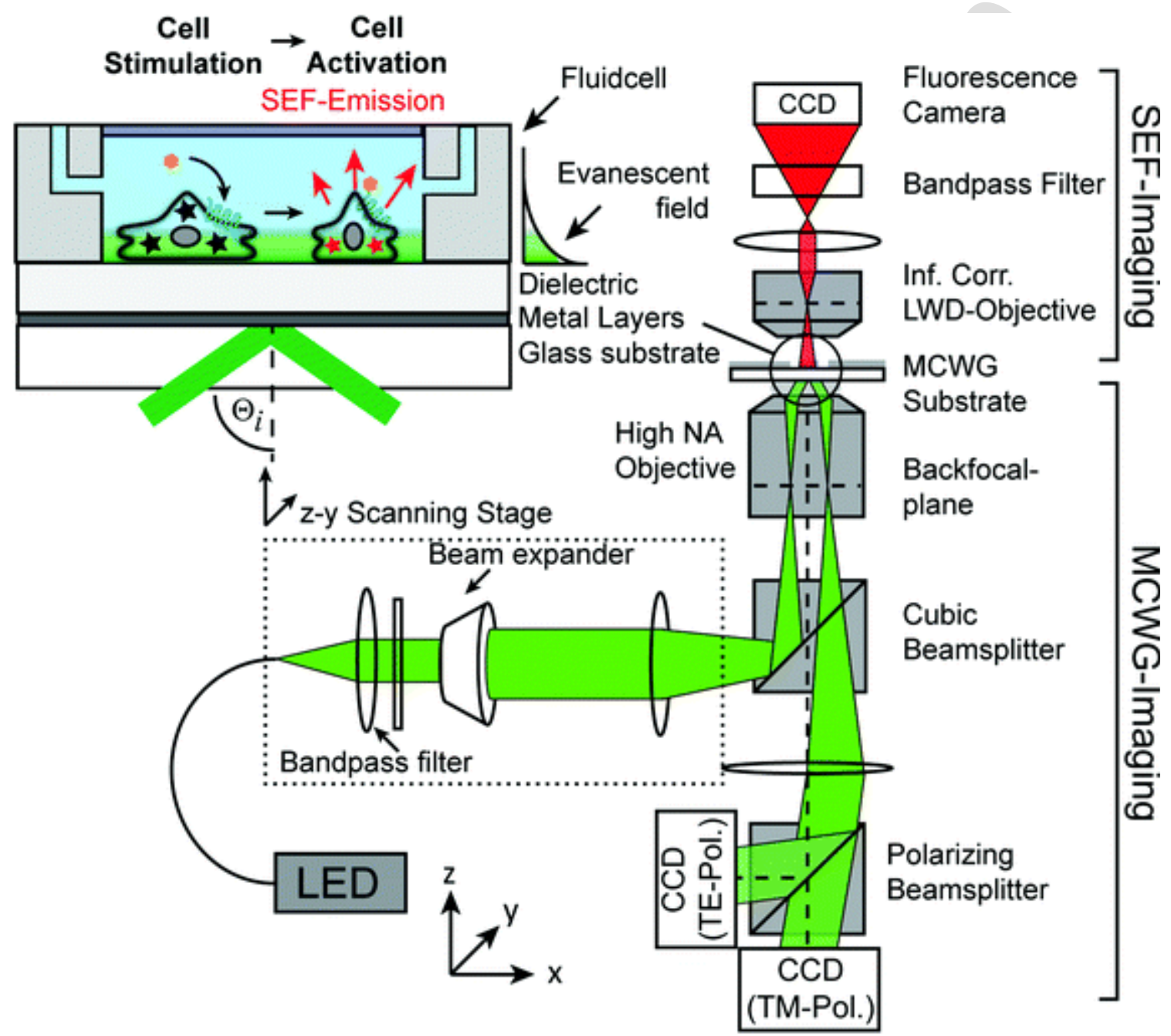

Fig. 1 Combined MCWG and SEF imaging platform. MCWG imaging is based on a highnumerical-aperture objective. SEF fluorescence emission is collected from the top by a long working distance objective.

The MCWG substrates are in fact planar optical waveguides fabricated on BK7 microscope slides consisting of a thin metal layer as the bottom cladding, a dielectric core, and a liquid sensing medium with the cells as the topmost cladding. ${ }^{13}$ Refractive index variations at the sensor surface 
caused by changes in cell morphology and/or internal cell reorganizations locally change the guided mode properties, which in turn result in reflected intensity changes recorded by the TMcomponent CCD camera. The relatively large refractive index differences $(1.355<\mathrm{n}<1.60)$ between the cellular organelles and the cytosol, as well as between the organelles themselves, $\underline{27}$ are easily resolvable in the MCWG images. Finally, the evanescent field in the liquid sensing medium excites fluorophores near the sensor surface, similarly to TIRF microscopy, yielding simultaneous MCWG and SEF imaging.

\subsection{Cell culture and fluorescence staining}

In a first series of experiments, an immortalized rat aortic vascular smooth muscle cell line (A7r5, ATCC, USA) was used to image the actin cytoskeleton components, where the cells were seeded on ethanol-sterilized MCWG substrates and F-actin was labeled with Alexa Fluor 532-phalloidin. Cells were maintained within selective Dulbecco's Modified Eagle's Medium (DMEM) supplemented with $10 \%$ heat-inactivated fetal bovine serum (FBS), $2.5 \mu \mathrm{g} \mathrm{m}{ }^{-1}$ amphotericin B, 50 $\mathrm{IU} \mathrm{ml^{-1 }}$ penicillin, $50 \mathrm{\mu g} \mathrm{ml}^{-1}$ streptomycin and supplemented with $1 \%$ non-essential amino acids. Post-fixation in 4\% paraformaldehyde (Sigma-Aldrich) for $10 \mathrm{~min}$, cells were washed twice with phosphate-buffered saline (PBS, Sigma-Aldrich) and incubated in tris-buffered saline (TBS, pH 8.1, Sigma-Aldrich) for $5 \mathrm{~min}$. After additional washing with PBS, the cell membrane was permeabilized using a $0.1 \%$ triton solution (Sigma-Aldrich) for 1 min followed by a thorough wash using PBS. Actin was stained by incubating the samples with Alexa Fluor 532-phalloidin (1:20 Thermo Fisher Scientific) for $20 \mathrm{~min}$. Samples were then washed and used immediately for imaging.

In a second series of experiments, intracellular GPCR signaling activity was studied in human embryonic kidney 293 cells (HEK 293, Qbiogene, QBI HEK-293A cells, USA), stably transfected to express the AT1R as described previously. ${ }^{28}$ Cells were maintained within selective DMEM supplemented with $10 \%$ heat-inactivated FBS, $0.4 \mathrm{mg} \mathrm{ml}^{-1} \mathrm{G}-418,2.5 \mu \mathrm{g} \mathrm{ml}^{-1}$ amphotericin $\mathrm{B}, 50$

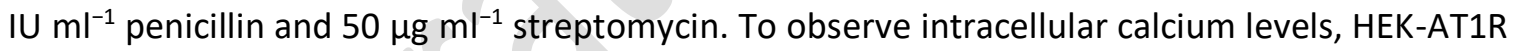
cells were seeded at a density of $5.75 \times 10^{5}$ on poly-L-lysine-coated sensor chips and grown to $60 \%$ confluence. Cells were washed twice with warm Hank's balanced salt solution (HBSS) and incubated with the calcium indicator Fluo-8 (5 $\mathrm{MM}$, Thermo Fisher Scientific) for $1 \mathrm{~h}$ at room temperature, washed twice and left for another $30 \mathrm{~min}$ in HBSS for de-esterification prior to an experiment. AT1R-activation was performed by exchanging the media in the sample chamber using a syringe pump (100 $\mu \mathrm{m} \mathrm{min}^{-1}$ for $8 \mathrm{~min}$ ) with HBSS buffer containing $100 \mathrm{nM}$ Angiotensin II (American Peptide). ROCK kinase activity was inhibited by pre-incubating the cells with Y27632 for $30 \mathrm{~min}(10 \mu \mathrm{M}$, Sigma-Aldrich) and the inhibitor was present throughout the experiment.

The microscopy platform relies on two separate optical systems to acquire the MCWG and SEF images from a sample, where the images reflect distinct types of information (MCWG: refractive index variations across the entire field of view, SEF: fluorescence intensity from specifically labeled cellular components). Although the fields of view and pixel correspondence between the two systems could be matched with appropriate optics, the depths of fields for both techniques are quite different and MCWG-based imaging has a characteristic planar spatial resolution imbalance. As a result, even with perfect pixel-correspondence, sub-cellular structural features may be difficult to correlate between the MCWG and SEF images. Indeed, this "correspondence problem" is common to all image-fusion systems, such as with PET (position emission 
tomography) and CT (X-ray computed tomography) for example. In general, the solution to this problem is to ensure that the samples contain clearly identifiable anatomical features in both images which can be used as spatial references. In the case of sub-cellular resolution imaging, this can be particularly challenging.

\section{Results and discussion}

\subsection{Actin cytoskeleton imaging by MCWG-SEF microscopy}

VSMCs exhibit a densely structured network of actin filaments $\frac{29}{}$ and are the mechanically active cell layer responsible for regulating vascular muscle tone and blood pressure. Fig. 2A shows a series of MCWG micrographs of a single VSMC adhered to the sensor surface taken at increasing angles of incidence $\left(\theta_{i}\right)$ to resonantly couple to a range of refractive indices in the sensing medium. At $64.17^{\circ}$ (Fig. 2A), resonant coupling to the fluid medium is achieved (darkest background indicating lowest reflectivity) and evanescent field intensity is maximized, where the dominant features in the MCWG micrograph are filamentous structures aligned with the long cellular axis. The greater probing depth provided by MCWG enables the visualization of structures in these cells that are consistent with actin filaments. $\underline{30}$ To confirm that these structures are indeed actin fibers, the high-affinity F-actin probe phalloidin-Alexa Fluor 532 was used to label the cell cytoskeleton and SEF micrographs were simultaneously recorded (Fig. 2B). As expected, the highest SEF signal intensity is achieved at $64.17^{\circ}$, an angle at which a good correlation between the elongated structures in the MCWG and SEF micrographs is observed. As explained above, spatial resolution in the imaging plane is anisotropic for label-free imaging modality. In our MCWG-SEF microscope, spatial resolution in the MCWG images is limited by diffraction limit of $\sim 0.18 \mu \mathrm{m}$ in the direction normal to mode propagation and to $\sim 5.0 \mu \mathrm{m}$ in the direction of mode propagation (black arrow in leftmost image in Fig. $2 \mathrm{~A}$ ) due to the finite attenuation length of the mode. $\frac{13}{.}$ This effect can also be seen in the second image from the left in Fig. $2 \mathrm{~A}\left(64.17^{\circ}\right)$ where the fibers aligned in the direction of propagation are clearly resolved (white arrow) while structures that are slightly off-axis appear partly blurred (white dotted arrow). Note since the fluorescent labels acts as point sources of light, the SEF signal is not subject to this effect (spatial resolution in the SEF images is isotropic and diffraction-limited in both axes). In addition, as observed by others with SPR, $\frac{31}{1}$ the presence of fluorophores with an absorption band that overlaps the excitation light spectrum locally modulates the (complex) refractive index in the sensing medium, acting as a contrast enhancement agent in the MCWG images. 

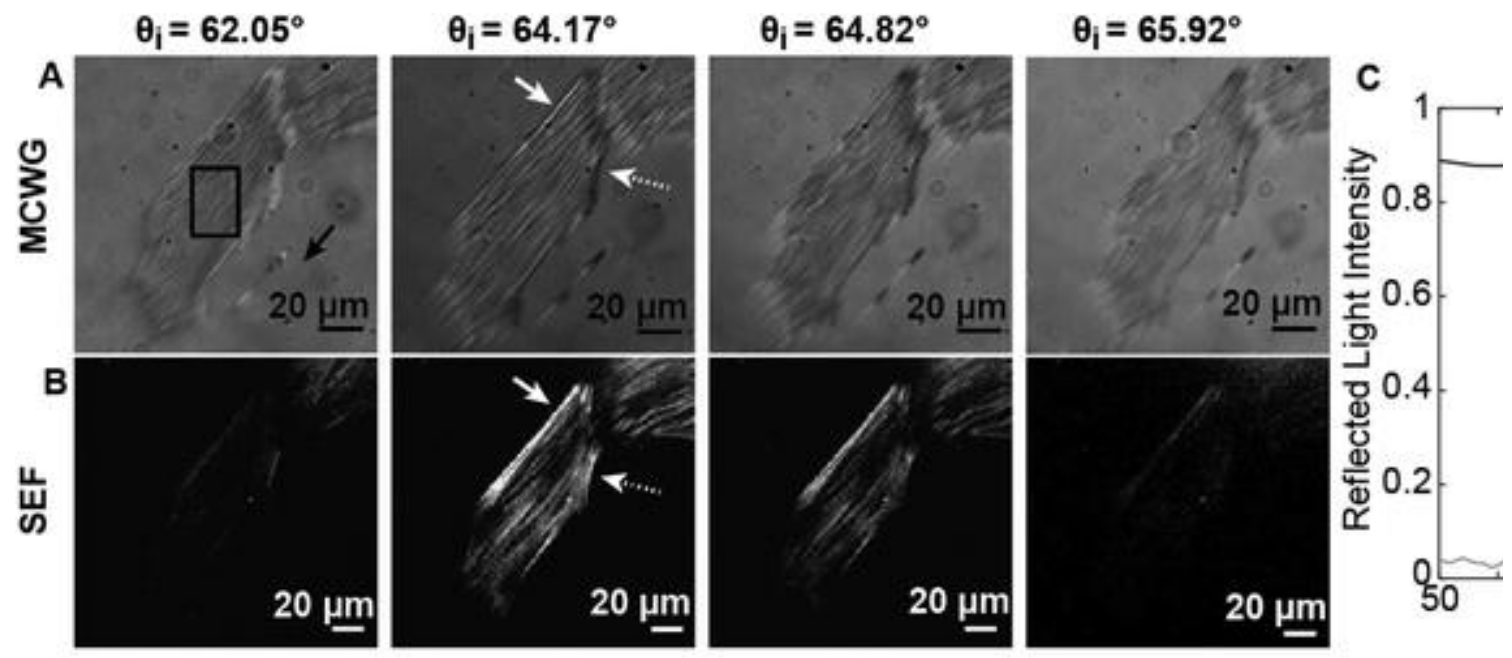

Fig. 2 Vascular smooth muscle cell simultaneously imaged by MCWG and SEF. (A) MCWG micrographs of a single VSMC taken at different angles of incidence. The black square in the leftmost image represents the location of ROI used to calculate average reflected intensity, while the black arrow indicates the direction of mode propagation; (B) SEF micrographs simultaneously taken at the same angles of incidence, F-actin was labeled with Alexa Fluor 532 Phalloidin; (C) plots of normalized average reflected light intensity within the ROIs in $A$ and overall average SEF intensity in B as a function of incidence angle.

Fig. 2C shows simultaneously acquired normalized plots of MCWG reflected light intensity (averaged within the ROI shown by the black square in Fig. 2A at $62.05^{\circ}$ ) and SEF intensity (overall image average) as a function of incidence angle. The minimum in MCWG reflected light intensity at $\theta_{i}=64.82^{\circ}$ is known as "critical coupling". At this angle, the electric field intensity at the sensor surface is very strong, yielding high fluorescence intensity (grey trace), as previously observed by our group and others for SPR. $\frac{13,23}{}$ As expected, maximum fluorescence intensity $\left(\theta_{i}=64.17^{\circ}\right)$ is slightly shifted from the minimum MCWG reflected light intensity since critical coupling occurs slightly off-resonance, where there is just enough out-coupled light from the guided mode to cancel the un-coupled incident light by destructive interference (in the SPR literature, $\frac{32}{2}$ this is referred to as the internal and re-radiation damping being balanced).

\subsection{AT1-induced cell activity analyzed by MCWG-SEF microscopy}

Cell-based, label-free technologies are now routinely used to monitor cellular responses associated with the activation of signaling pathways in cell populations to provide insight into

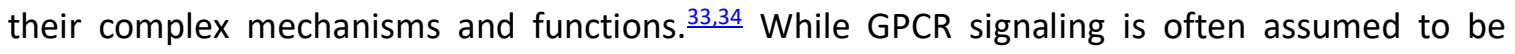
homogeneous within a cell population, receptor expression levels and variations in individual cell phenotypes can alter their overall response profile and lead to heterogeneous population responses. $\frac{35,36}{3}$ To demonstrate the possibility of assessing such population-dependent behavior, we used a HEK293 cell model stably transfected with AT1R. $\underline{37}$ In this cell model, two major signaling cascades are involved following receptor activation by Angll, namely the $\mathrm{G}_{\mathrm{q}}$-protein dependent signaling cascade leading to intracellular calcium mobilization and the 
$\mathrm{G}_{12 / 13} / \mathrm{Rho} / \mathrm{ROCK}$ pathways. These pathways are known to be involved in both the regulation of the cytoskeleton organization and actin-myosin-dependent contractile activity. $\frac{38,39}{}$

We used the MCWG-SEF microscopy system to follow intracellular calcium levels with the fluorescent probe Fluo8 (SEF imaging) while simultaneously monitoring the overall cellular morphological response (MCWG imaging) resulting from AT1 receptor activation by Angll. Fig. 3 shows a time series sample of eight individual cells imaged with MCWG (left image column) and SEF (right image column). As expected, the cell morphology in the MCWG and SEF images (Fig. $3 \mathrm{~A}$ and G) appear slightly different since the MCWG signal in our configuration originates from nonspecific refractive index differences within a $\sim 0.5 \mu \mathrm{m}$ thick sensing volume $\frac{13}{3}$ whereas the SEF signal originates from the presence of a calcium indicator distributed within a $\sim 1 \mu \mathrm{m}$ thick volume above the sensor surface. MCWG micrographs B-E are color-coded maps of percentage reflectance difference relative to the reference (Fig. 3A), where the blue end of the spectrum indicates a decrease, the red end of the spectrum indicates an increase, and green indicates no change. Following Angll stimulation at $0 \mathrm{~min}$, a burst of intracellular calcium is observed within the first $5 \mathrm{~min}$ in the SEF images and quantified in the average fluorescence intensity plots, confirming receptor activation and functionality of the signaling pathway in this cell model. The burst of intracellular calcium is followed by the appearance of localized decreases in MCWG reflectance (blue regions) attributable to a reduction in cellular mass density within the effective sensing range of the evanescent field, consistent with increased acto-myosin contractile activity, $\underline{\underline{21}, 28}$ where the minimum MCWG average signal intensity occurs at $\sim 6.5 \mathrm{~min}$. The initial burst in intracellular calcium level is then followed by a recovery phase were calcium levels return to near basal levels after $15 \mathrm{~min}$ to $35 \mathrm{~min}$ for most cells (Fig. 3I and J), as generally observed for receptor coupled to the calcium pathway. In contrast, the MCWG micrographs (Fig. 3D and E) show a distinctly heterogeneous behavior within the cells with both localized increases and decreases in signal levels, indicating a re-modeling of intracellular structures associated with cell activation $\underline{14}$ and possibly as a result of AT1 receptor-signaling activity. $\underline{.21,40}$ 

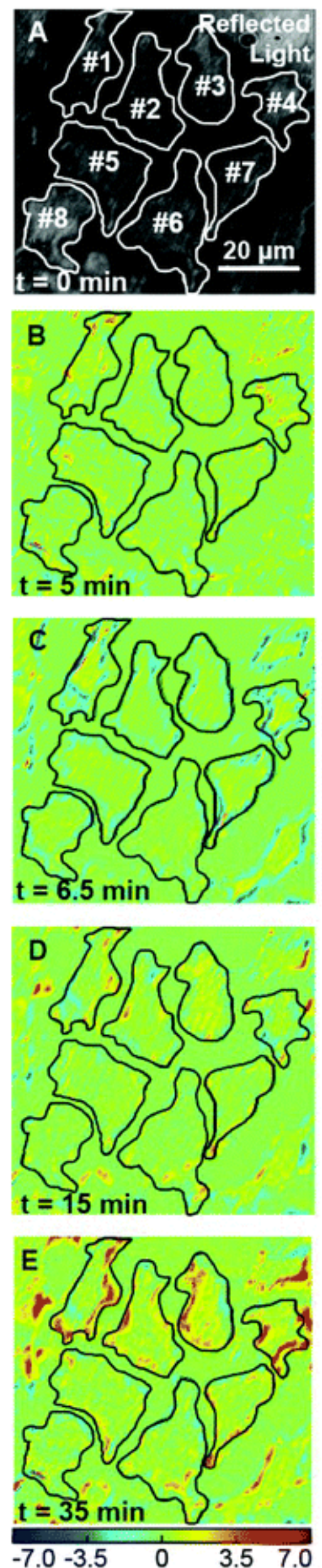

Reflectance Vairation [\%]

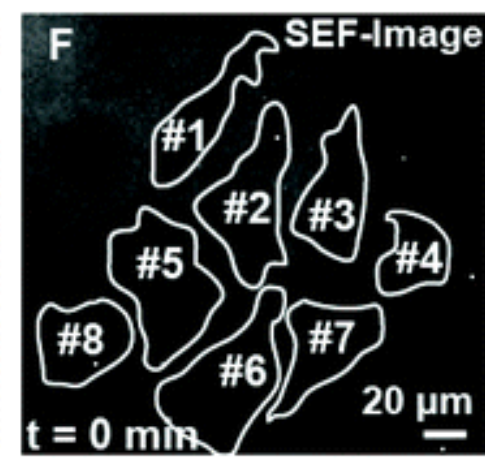

K HBSS Exchange $\frac{}{\text { Phase I }}$
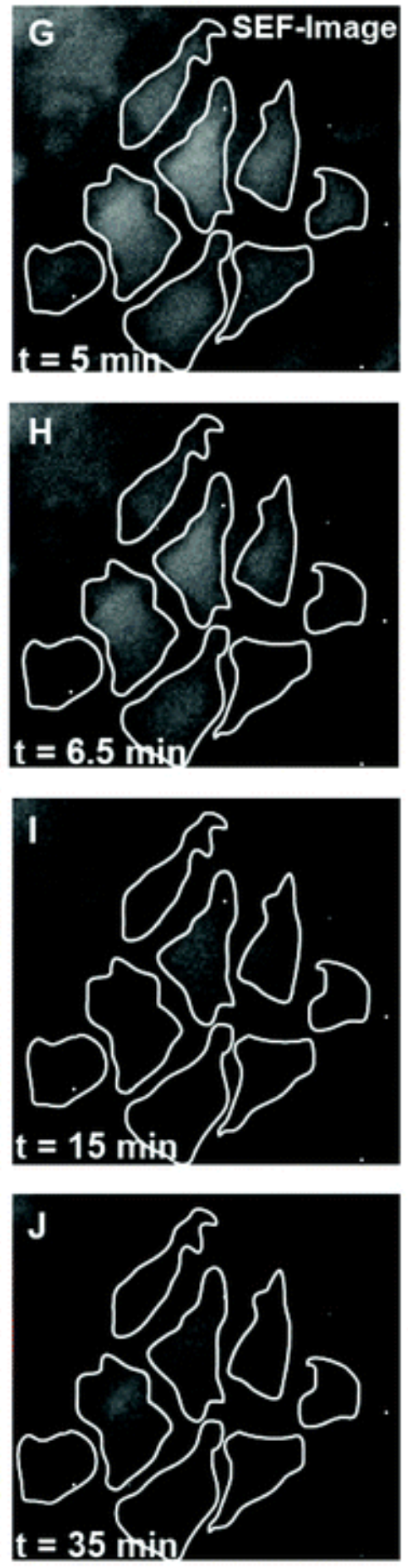

Cell \#4

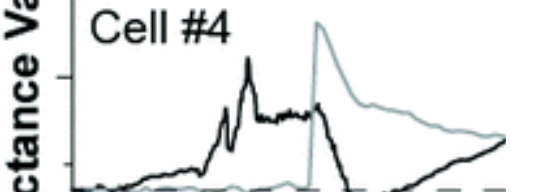

产
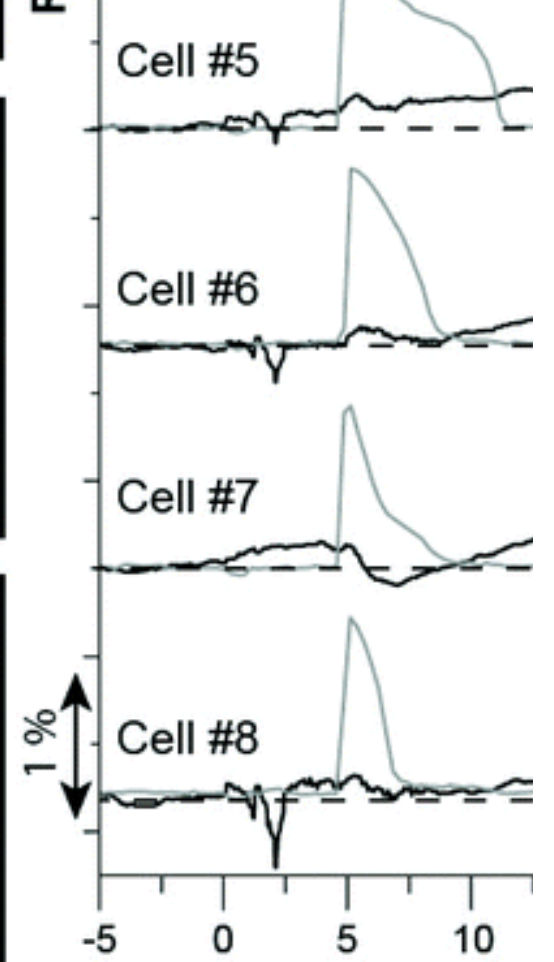

$\operatorname{Tin}$ 
Fig. 3 Simultaneous monitoring of intracellular calcium by SEF imaging and cellular morphological response by MCWG imaging (A) reflectance micrograph of 8 individual HEK-AT1 cells; $(B-E)$ reflectance variation micrographs showing local changes in reflectance following stimulation with $100 \mathrm{nM}$ Angll at $\mathrm{t}=0 \mathrm{~min}$ (blue end of the spectrum indicates a decrease, red end of the spectrum indicates an increase, green indicates no change); (F-J) simultaneous SEF micrographs, where cells were loaded with calcium indicator Fluo-8; (K) plots of the full-time sequence of average MCWG reflected intensity and SEF average intensity, normalized by cell surface area.

The plots in Fig. 3K show the time sequences of averaged MCWG reflected intensity and SEF average intensity, with both signals normalized by cell surface area as presented in Fig. 3A and F. Note that some cells show a certain degree of signal variation during the baseline phase as well as during the onset of the media exchange. Such drifts could be associated with minute changes in the fluid cell temperature or with flow induced effects from the media exchange. The plots show a biphasic response in both the SEF and the MCWG time profiles. Phase I is associated with the initial burst of intracellular calcium following stimulation of the cells with Angll (5 min), confirming the receptor activation and subsequent $\mathrm{G}_{\mathrm{q}}$ signaling known to mobilize calcium from the endoplasmic reticulum. $\stackrel{28}{ }$ Phase $I I$ of the reflectance signal is associated in time to the recovery of the intracellular calcium levels for all cells. Intriguingly, these oscillations in calcium levels were not found to be associated to the MCWG signal, indicating that they do not induce significant morphological changes or cell body contraction in this cell model. It should be noted here that oscillating calcium signals are a well-known phenomenon and provide a digital signal to downstream effectors, involved in the regulation of gene expression, a process that normally occurs over several hours. $\underline{41}$

As expected, the SEF and MCWG data are strongly correlated, confirming the receptor-dependent cell signaling response origin of the MCWG label-free signal. However, significant differences can also be observed when comparing individual responses. During phase I, though all cells showed an intracellular calcium burst (indication of AT1R functionality), only cells \#1-4 and 7 showed a subsequent marked decrease in average MCWG reflectance. During phase II, cells \#1, \#5, \#7 and \#8 exhibited oscillations in their intracellular calcium levels with varying periodicity $(116 \mathrm{~s}, 230 \mathrm{~s}$, $265 \mathrm{~s}$ and $173 \mathrm{~s}$, respectively), while cells \#2, \#3 and \#4 showed sustained elevated calcium levels. The absence of such distinct features within the MCWG signal further demonstrates that changes in the intracellular calcium levels seem to not interfere with the observed MCWG signal, which has also been demonstrated for other label-free detection modalities. $\underline{21}$ These observations indicate that the $\mathrm{G}_{\mathrm{q}}$-dependent calcium signal alone is be insufficient to explain the first phase of the MCWG signal, indicating that other pathways may be required for the phase I of the MCWG signal.

\subsection{Role of ROCK and $\mathrm{G}_{12 / 13}$ during $A T 1$ signaling and link to cellular activity}

In addition to the calcium-dependent $\mathrm{G}_{\mathrm{q}}$ signaling cascade, AT1R is also coupled to a calciumindependent $G_{12 / 13}$ signaling pathway. $\frac{39}{3}$ The activation of this pathway ultimately leads to

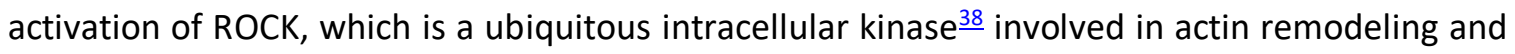
the regulation of actomyosin contractile activity via myosin light chain (MLC) phosphorylation and inhibition of the myosin light chain phosphatase (MLCP). To test the involvement of $\mathrm{G}_{12 / 13}$ 
signaling and thus the involvement of ROCK in the measured cellular response, HEK293-AT1R cells were treated with the ROCK inhibitor Y-27632 prior to activation with AngII.

Fig. 4A-J show MCWG (A: reference, B-E: differences) and SEF micrographs from the experiment, alongside plots of cell-specific average MCWG and SEF responses for the time series normalized by cell area (Fig. 4K). As expected, the MCWG data do not show a signal decrease (phase I) following AT1 activation, confirming the robust suppression of cell signaling activity through ROCK inhibition (Fig. 4C). The phase II in the MCWG signal response nevertheless appears not to be impacted as indicated by the appearance of large yellow and red areas in the MCWG micrographs (Fig. 4D and E). The SEF data again shows a robust release of intracellular calcium, as expected from AT1 activation (Fig. 4G-J). When comparing the experimental time series (Fig. 4K), interestingly despite the absence of a Phase I response in the MCWG data, all cells nevertheless show a steady increase in average MCWG response during Phase II suggesting a distinct $\mathrm{G}_{12 / 13^{-}}$ independent signaling mechanism for Phase II compared to the previous experiment due to additional unidentified component. The SEF time series shows again a biphasic response profile where all cells respond with a transient release of intracellular calcium shortly after Angll stimulation as expected from AT1 activation. Overall, these results indicate that $\mathrm{G}_{12 / 13}$ signaling may be the major signaling pathway responsible for Phase $I$ and is consistent with previous population-based findings using SPR spectroscopy in which ROCK inhibition, but not MLCK inhibition, associated with $G_{q}$ signaling could successfully suppress Phase I contractile response. $\underline{\underline{21}}$ 

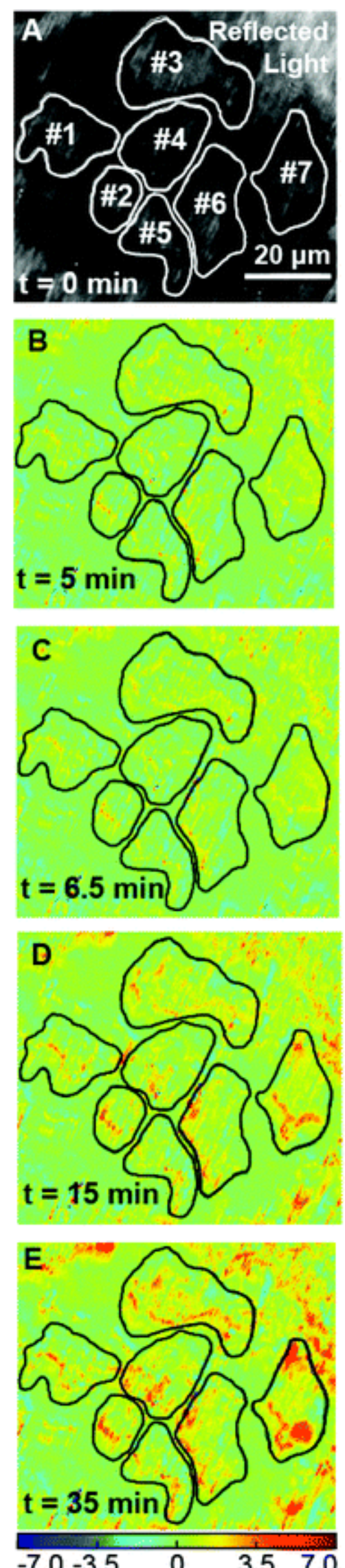

Reflectance Vairation [\%]
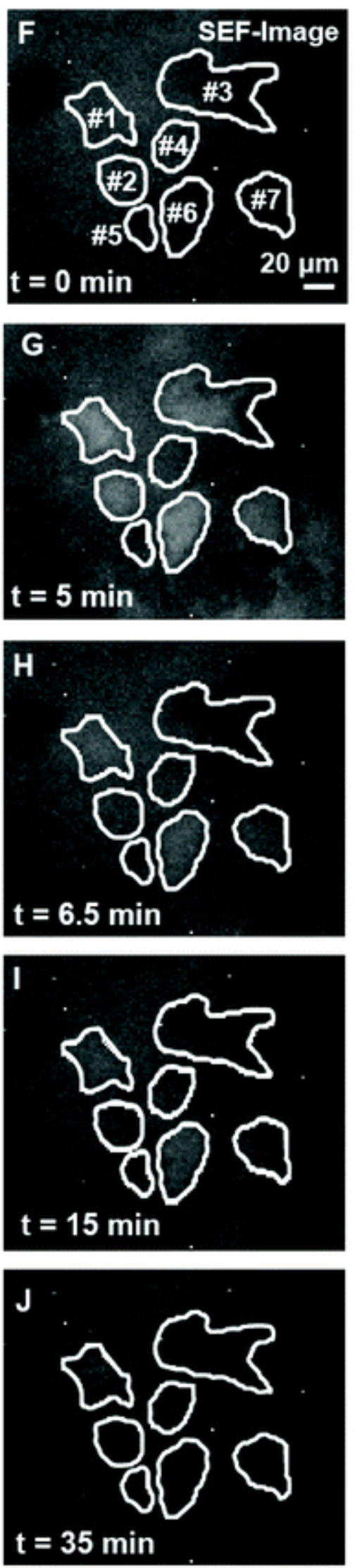
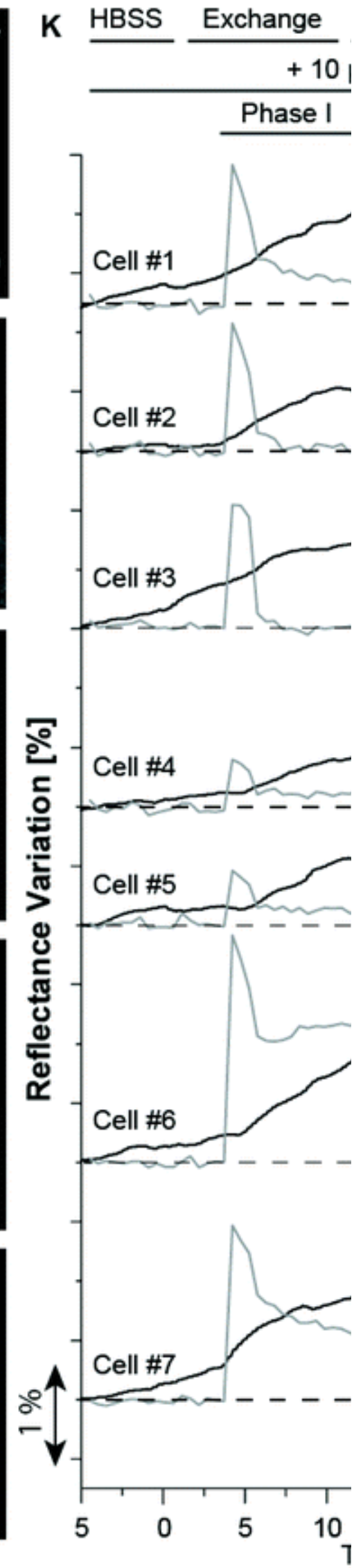
Fig. 4 Impact on ROCK inhibition during AT1 analyzed by MCWG and SEF microscopy. (A) Reflectance micrograph of individual HEK-AT1R cells; (B-E) reflectance variation micrographs showing local changes in reflectance after pretreatment with Y-27632 and stimulation with 100 nM AngII; (F-J) SEF micrographs of the same cells, loaded with calcium indicator Fluo-8; (K) plots of cell-specific average MCWG and SEF responses for the full time series normalized by cell area.

\section{Conclusion}

In this work, we demonstrated a combined MCWG and SEF microscopy system for dual mode imaging and signal analysis of receptor-dependent cell signaling activity. We demonstrated the capabilities of this system in experiments designed to (1) identify the specific structural components of the cytoskeleton imaged by MCWG and SEF, and (2) provide insight into intracellular signaling following GPCR activation in living cells. In the latter case, SEF imaging revealed the intracellular $\mathrm{G}_{\mathrm{q}}$-dependent calcium mobilization while simultaneous $M C W G$ imaging showed the distinct individual cell response profiles to Angll-mediated AT1R activation. A strong temporal correlation was observed between the MCWG label-free signal and the labelled calcium signal from individual cells, confirming the origin of the MCWG label-free signal as a receptordependent cell signaling response. Interestingly, a discrepancy between the two signals observed for some cells suggest that the cell signaling activity measured through the MCWG signal channel is not necessarily dependent on calcium mobilization. This type of observation is only possible through a comparative analysis of individual cell heterogeneity present in the two distinct response profiles. Using a selective $\mathrm{G}_{12 / 13}$ /Rho/ROCK signaling inhibitor, we determined that this pathway is required for the phase I response observed with MCWG imaging. Interestingly, the second phase of the response profile was not impacted by $\mathrm{G}_{12 / 13} /$ Rho/ROCK signaling inhibition, suggesting that other pathways such as $\mathrm{G}_{\mathrm{q}}$-dependent pathway may be required for this later phase. While other signaling cascades could also be involved in AT1 signaling, this work shows how individual cell assessment using MCWG and SEF together with pharmacological tools and fluorescent probes targeting specific cell signaling pathways can enables the identification of key contributors to a given cellular response. Thus, this system should find applications in drug screening, signaling cascade profiling, and in the study of fundamental cellular mechanisms.

\section{Conflicts of interest}

There are no conflicts to declare.

\section{Acknowledgements}

This work was supported by the Fonds de recherche du Québec - Nature et technologies (FRQNT: MG, PGC, TS), the Natural sciences and engineering research council of Canada (NSERC: MG, PGC, $\mathrm{MC}$ ), and the Canadian Institutes of Health Research (CIHR) under the Collaborative Health Research Program (MG, PGC). LN2 is an international research laboratory co-managed in Canada by University of Sherbrooke and in France by CNRS, Université de Lyon (Centrale Lyon, INSA Lyon et CPE Lyon), and Université Grenoble-Alpes; LN2 is funded in Québec by the FRQNT. TS was also supported by the COMET K1 center Austrian Smart Systems Integration Research (ASSIC). The 
COMET Competence Centers for Excellent Technologies Program is supported by BMVIT, BMWFW and the federal provinces of Carinthia and Styria of Austria.

\section{References}

1. G. K. Owens, Physiol. Rev., 1995, 75, 487-517 CrossRef CAS PubMed .

2. K. Palczewski, T. Kumasaka, T. Hori, C. A. Behnke, H. Motoshima, B. A. Fox, I. Le Trong, D. C. Teller, T. Okada, R. E. Stenkamp, M. Yamamoto and M. Miyano, Science, 2000, 289, 739-745 CrossRef CAS PubMed.

3. Y. Fang, A. M. Ferrie, N. H. Fontaine, J. Mauro and J. Balakrishnan, Biophys. J., 2006, 91, 1925-1940 CrossRef CAS PubMed.

4. C. Tiruppathi, A. B. Malik, P. J. Del Vecchio, C. R. Keese and I. Giaever, Proc. Natl. Acad. Sci. U. S. A., 1992, 89, 7919-7923 CrossRef CAS.

5. V. Chabot, C. M. Cuerrier, E. Escher, V. Aimez, M. Grandbois and P. G. Charette, Biosens. Bioelectron., 2009, 24, 1667-1673 CrossRef CAS PubMed.

6. Y. Yanase, H. Suzuki, T. Tsutsui, T. Hiragun, Y. Kameyoshi and M. Hide, Biosens. Bioelectron., 2007, 22, 1081-1086 CrossRef CAS PubMed.

7. M. M. A. Jamil, M. Youseffi, S. T. Britland, S. Liu, C. W. See, M. G. Somekh and M. C. T. Denyer, Image, 2007, 15, 334-337 Search PubMed.

8. A. W. Peterson, M. Halter, A. Tona and A. L. Plant, BMC Cell Biol., 2014, 15, 35 CrossRef PubMed.

9. W. Wang, S. Wang, Q. Liu, J. Wu and N. Tao, Langmuir, 2012, 28, 1337313379 CrossRef CAS PubMed.

10. Y. Yanase, T. Hiragun, S. Kaneko, H. J. Gould, M. W. Greaves and M. Hide, Biosens. Bioelectron., 2010, 26, 674-681 CrossRef CAS PubMed.

11. K. Giebel, C. Bechinger, S. Herminghaus, M. Riedel, P. Leiderer, U. Weiland and M. Bastmeyer, Biophys. J., 1999, 76, 509-516 CrossRef CAS PubMed.

12. N. Skivesen, R. Horvath and H. C. Pedersen, Sens. Actuators, B, 2005, 106, 668676 CrossRef CAS.

13. T. Söllradl, F. A. Banville, V. Chabot, M. Canva, M. Grandbois and P. G. Charette, Opt. Express, 2017, 25, 1666 CrossRef PubMed.

14. T. Söllradl, F. A. Banville, U. Froehlich, M. Canva, P. G. Charette and M. Grandbois, Biosens. Bioelectron., 2018, 100, 429-436 CrossRef PubMed.

15. J. Lu, Y. Yang, W. Wang, J. Li, N. Tao and S. Wang, Anal. Chem., 2016, 6b02677 Search PubMed.

16. L. Berguiga, L. Streppa, E. Boyer-Provera, C. Martinez-Torres, L. Schaeffer, J. Elezgaray, A. Arneodo and F. Argoul, Appl. Opt., 2016, 55, 1216 CrossRef PubMed . 
17. F. Banville, T. Söllradl, P.-J. Zermatten, M. Grandbois and P. G. Charette, Opt. Lett., 2015, 40, 1165-1168 CrossRef CAS PubMed.

18. E. M. Yeatman, Biosens. Bioelectron., 1996, 11, 635-649 CrossRef CAS.

19. A. W. Peterson, M. Halter, A. Tona, K. Bhadriraju and A. L. Plant, BMC Cell Biol., 2009, 10, 16 CrossRef PubMed.

20. R.-Y. He, C.-Y. Lin, Y.-D. Su, K.-C. Chiu, N.-S. Chang, H.-L. Wu and S.-J. Chen, Opt. Express, 2010, 18, 3649-3659 CrossRef CAS PubMed.

21. C. M. Cuerrier, V. Chabot, S. Vigneux, V. Aimez, E. Escher, F. Gobeil, P. G. Charette and M. Grandbois, Cell. Mol. Bioeng., 2008, 1, 229-239 CrossRef CAS PubMed.

22. V. Chabot, Y. Miron, P. G. Charette and M. Grandbois, Biosens. Bioelectron., 2013, 50, 125-131 CrossRef CAS PubMed.

23. T. Liebermann and W. Knoll, Colloids Surf., A, 2000, 171, 115-130 CrossRef CAS .

24. D. Yao, F. Yu, J. Kim, J. Scholz, P. E. Nielsen, E.-K. Sinner and W. Knoll, Nucleic Acids Res., 2004, 32, e177 CrossRef PubMed.

25. R.-Y. He, Y.-D. Su, K.-C. Cho, C.-Y. Lin, N.-S. Chang, C.-H. Chang and S.-J. Chen, Opt. Express, 2009, 17, 5987 CrossRef CAS PubMed.

26. R.-Y. He, G.-L. Chang, H.-L. Wu, C.-H. Lin, K.-C. Chiu, Y.-D. Su and S.-J. Chen, Opt. Express, 2006, 14, 9307 CrossRef PubMed.

27. Y. Liu, L. K. Chin, W. Ser, H. Chen, C.-M. Hsieh, C.-H. Lee, K.-B. Sung, T. C. Ayi, P. H. Yap, B. Liedberg, K. Wang, T. Bourouina and Y. Leprince-Wang, Lab Chip, 2015, 16, 634-644 RSC.

28. M. Auger-Messier, G. Arguin, B. Chaloux, R. Leduc, E. Escher and G. Guillemette, Mol. Endocrinol., 2004, 18, 2967-2980 CrossRef CAS PubMed.

29. G. K. Owens, M. S. Kumar and B. R. Wamhoff, Physiol. Rev., 2004, 84, 767801 CrossRef CAS PubMed.

30. P. Kanchanawong, G. Shtengel, A. M. Pasapera, E. B. Ramko, M. W. Davidson, H. F. Hess and C. M. Waterman, Nature, 2010, 468, 580-584 CrossRef CAS PubMed.

31. M. Nakkach, P. Lecaruyer, F. Bardin, J. Sakly, Z. Ben Lakhdar and M. Canva, Appl. Opt., 2008, 47, 6177-6182 CrossRef PubMed.

32. H. Raether, Surface plasmons on smooth and rough surfaces and on gratings, Springer, 1988 Search PubMed.

33. R. Schröder, N. Janssen, J. Schmidt, A. Kebig, N. Merten, S. Hennen, A. Müller, S. Blättermann, M. Mohr-Andrä, S. Zahn, J. Wenzel, N. J. Smith, J. Gomeza, C. Drewke, G. Milligan, K. Mohr and E. Kostenis, Nat. Biotechnol., 2010, 28, 943-949 CrossRef PubMed.

34. C. W. Scott and M. F. Peters, Drug Discovery Today, 2010, 15, 704716 CrossRef $\underline{\text { CAS PubMed. }}$. 
35. G. Nickenig, A. Sachinidis, F. Michaelsen, M. Bohm, S. Seewald and H. Vetter, Circulation, 1997, 95, 473-478 CrossRef CAS PubMed.

36. M. E. Wechsler, E. Lehman, S. C. Lazarus, R. F. Lemanske, H. A. Boushey, A. Deykin, J. V. Fahy, C. A. Sorkness, V. M. Chinchilli, T. J. Craig, E. DiMango, M. Kraft, F. Leone, R. J. Martin, S. P. Peters, S. J. Szefler, W. Liu and E. Israel, Am. J. Respir. Crit. Care Med., 2006, 173, 519-526 CrossRef CAS PubMed.

37. W. L. Kaufman, I. Kocman, V. Agrawal, H.-P. Rahn, D. Besser and M. Gossen, Nucleic Acids Res., 2008, 36, e111-e111 CrossRef PubMed.

38. J. K. Park, S. O. Lee, Y. G. Kim, S. H. Kim, G. Y. Koh and K. W. Cho, Int. J. Impotence Res., 2002, 14, 472-477 CrossRef CAS PubMed.

39. D. A. Emmert, J. A. Fee, Z. M. Goeckeler, J. M. Grojean, T. Wakatsuki, E. L. Elson, B. P. Herring, P. J. Gallagher and R. B. Wysolmerski, Am. J. Physiol.: Cell Physiol., 2003, 286, C8C21 CrossRef PubMed.

40. C. M. Cuerrier, M. Benoit, G. Guillemette, F. Gobeil Jr. and M. Grandbois, Pflügers Arch. Eur. J. Physiol., 2009, 457, 1361-1372 CrossRef CAS PubMed.

41. R. E. Dolmetsch, K. Xu and R. S. Lewis, Nature, 1998, 392, 933-936 CrossRef $\underline{\text { CAS }}$ PubMed. 